\title{
Does Complexity Equal Anything?
}

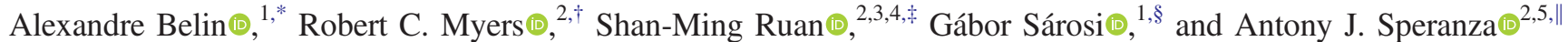 \\ ${ }^{1}$ CERN, Theory Division, 1 Esplanade des Particules, Geneva 23, CH-1211, Switzerland \\ ${ }^{2}$ Perimeter Institute for Theoretical Physics, Waterloo, Ontario N2L 2Y5, Canada \\ ${ }^{3}$ Department of Physics and Astronomy, University of Waterloo, Waterloo, Ontario N2L 3G1, Canada \\ ${ }^{4}$ Yukawa Institute for Theoretical Physics, Kyoto University, Kitashirakawa Oiwakecho, Sakyo-ku, Kyoto 606-8502, Japan \\ ${ }^{5}$ Department of Physics, University of Illinois, Urbana-Champaign, Urbana, Illinois 61801, USA
}

(Received 18 November 2021; accepted 3 February 2022; published 23 February 2022)

\begin{abstract}
We present a new infinite class of gravitational observables in asymptotically anti-de Sitter space living on codimension-one slices of the geometry, the most famous of which is the volume of the maximal slice. We show that these observables display universal features for the thermofield-double state: they grow linearly in time at late times and reproduce the switchback effect in shock wave geometries. We argue that any member of this class of observables is an equally viable candidate as the extremal volume for a gravitational dual of complexity.
\end{abstract}

DOI: 10.1103/PhysRevLett.128.081602

Introduction.-Complexity theory aims to quantify how difficult it is to perform a chosen task using a set of simple operations. In quantum complexity theory, one implements the desired operation by constructing a quantum circuit with simple unitary gates. An important aspect to keep in mind is that in complexity theory, the interest is always on the scaling of the complexity with the "size" of the problem (e.g., the dimensionality of the Hilbert space in quantum complexity). Extracting a precise result for the complexity is often too complicated, and this number is expected to be highly sensitive, e.g., to the choice of allowed simple operations, while the scaling is a robust property of the problem class.

Quantum complexity has recently triggered much interest in the context of black holes and holography, as a new twist in the ongoing effort connecting quantum information theory to quantum gravity. The length of the wormhole for a two-sided anti-de Sitter (AdS) black hole grows linearly in time at late times, and continues growing far beyond times at which entanglement entropies have thermalized [1]. This suggests that a new quantum information measure is needed to encode the growth of the wormhole, and several holographic proposals were made relating it to quantum complexity, e.g., complexity has been proposed to be dual to the volume of the maximal slice (CV proposal) [2], the action of the Wheeler-de Witt patch (CA proposal) [3] or the spacetime volume of the Wheeler-de Witt patch (CV2.0 proposal) [4].

Published by the American Physical Society under the terms of the Creative Commons Attribution 4.0 International license. Further distribution of this work must maintain attribution to the author(s) and the published article's title, journal citation, and DOI. Funded by SCOAP ${ }^{3}$.
It is important to emphasize that the formulation of all three of the above proposals is ambiguous. For CV and CV2.0, this comes from an additional length scale needed to obtain a dimensionless number out of the volume. For CA, it results from ambiguities in the boundary terms on null slices [5]. However, rather than a shortcoming, this ambiguity can be seen as a feature of holographic complexity, as it connects nicely with the ambiguities arising in complexity theory, e.g., the choice of a gate set. Therefore, a gravitational dual for complexity should reflect these conventional ambiguities.

In this Letter, we explore this idea and show that, in fact, there is an infinite class of gravitational observables defined in a diffeomorphism-invariant way that display universal features and hence are equally viable candidates for a gravitational dual of complexity as the volume or the action. These observables are defined on codimensionone regions of the geometry as

$$
O_{F_{1}, \Sigma_{F_{2}}}\left(\Sigma_{\mathrm{CFT}}\right)=\frac{1}{G_{N} L} \int_{\Sigma_{F_{2}}} d^{d} \sigma \sqrt{h} F_{1}\left(g_{\mu \nu} ; X^{\mu}\right)
$$

where $F_{1}$ is a scalar function of the background metric $g_{\mu \nu}$ and of an embedding $X^{\mu}\left(\sigma^{a}\right)$ of the codimension-one surface $\Sigma_{F_{2}}$. Asymptotically $\Sigma_{F_{2}}$ is fixed by the boundary condition $\partial \Sigma_{F_{2}}=\Sigma_{\mathrm{CFT}}$. In this Letter, we will focus on the case where $\Sigma_{\mathrm{CFT}}$ is a constant time slice in the boundary CFT, and so we often write $O_{F_{1}, \Sigma_{F_{2}}}(\tau)$ ( $\tau$ is the CFT time). For such quantities to be diffeomorphism invariant, the bulk slice must be defined in a coordinate independent way. We determine this slice by requiring that it extremizes a particular scalar functional $F_{2}$, i.e., 


$$
\delta_{X}\left(\int_{\Sigma} d^{d} \sigma \sqrt{h} F_{2}\left(g_{\mu \nu} ; X^{\mu}\right)\right)=0 .
$$

Note that generally the scalar functions $F_{1}$ and $F_{2}$ need not coincide. For $F_{1}=F_{2}=1$, this prescription yields the extremal volume appearing in the CV proposal. Allowing for more general functions gives an infinite family of new observables.

A class of such generalized functionals has appeared previously in the context of holographic complexity for higher curvature theories of gravity [6-8], in which the volume functional is corrected with higher curvature contributions. The present work emphasizes that such functionals also serve as good measures of holographic complexity in standard general relativity (or potentially any diffeomorphism-invariant gravitational theory), since they are examples of observables $O_{F_{1}, \Sigma_{F_{2}}}$ with $F_{1}=F_{2}$.

Summary of results.-We show that for a large subset of the class of functionals $O_{F_{1}, \Sigma_{F_{2}}}$, the two following universal properties hold when probing the thermofield double state: (1) Observables grow linearly with time at late times

$$
\lim _{\tau \rightarrow \infty} O_{F_{1}, \Sigma_{F_{2}}}(\tau) \sim P_{\infty} \tau
$$

In the limit of large temperature, the constant $P_{\infty}$ is proportional to the mass. (2) Observables exhibit the switchback effect [9], a universal time delay in response to perturbations described by particles falling into the dual black hole. Properties 1 and 2 are expected to be displayed universally by any definition of quantum complexity and are held as the main evidence for the $\mathrm{CV}$ and CA proposals. The linear growth property is a consequence of viewing the time evolution operator $\exp (-i H \tau)$ as a quantum circuit, whose size scales as $\propto \tau$. On the other hand, the switchback effect is an imprint of the butterfly effect on complexity growth. We can insert an operator at some time $t$ in the past which corresponds to acting with $\exp (-i H t) W \exp (i H t)$ on our state. If we do not add any operator $W$, the backward and forward time evolution exactly cancel. With the operator, the cancellation is disturbed but still occurs approximately for some time, before the effect of the operator grows large due to the butterfly effect. This logic suggests the size of the circuit implementing $\exp (-i H t) W \exp (i H t)$ for large $t$ is $\sim 2 P_{\infty}\left(t-t_{*}\right)$, where $t_{*}$ is the scrambling time [9].

The universality displayed by the class of observables (1) leads us to conclude that any of them are equally good candidates for a gravitational dual of complexity. This nicely parallels the expected ambiguities for quantum complexity, noted above, where a precise definition of the complexity depends on many choices. Hence the precise value of the complexity is unimportant, whereas the scaling (here, the time dependence) is universal.
Observables with $F_{1}=F_{2}$. - The quantities (1) provide a huge class of diff-invariant observables in AdS which can probe a plethora of states, ranging from small perturbations of AdS to black holes. In this Letter, we focus on the eternal planar black hole, which corresponds to two decoupled CFTs on planar (i.e., $\mathbb{R}^{d-1}$ ) spatial slices entangled in the thermofield double (TFD) state

$$
\left|\psi_{\mathrm{TFD}}(\tau)\right\rangle=\sum_{E_{n}} e^{-\beta E_{n} / 2-i E_{n} \tau}|n\rangle_{L} \otimes|n\rangle_{R}
$$

The spacetime dual to this state is described in EddingtonFinkelstein coordinates by the metric

$$
d s^{2}=-f(r) d v^{2}+2 d v d r+\frac{r^{2}}{L^{2}} d \vec{x}^{2},
$$

where $f(r)=\left(r^{2} / L^{2}\right)\left[1-\left(r_{h}^{d} / r^{d}\right)\right]$, and we use the infalling coordinate $v=t+r_{*}(r)$ with $r_{*}(r)=-\int_{r}^{\infty}\left[d r^{\prime} / f\left(r^{\prime}\right)\right]$ to cover the black hole interior. The spacetime describes the time evolution of the CFT state living at time $t_{R}=$ $t_{L}=\tau / 2$ (see Fig. 1).

We will start by considering observables with $F_{1}=F_{2}$, such that the slice on which the functional is evaluated extremizes the functional itself. We will call these observables $\mathcal{C}_{\text {gen }}$, as they generalize the $\mathrm{CV}$ proposal. To probe the state (4), we must anchor the surface on the CFT slice $\Sigma_{\tau}$, i.e., the boundary of the hypersurface $\Sigma(\tau)$. Thus,

$\mathcal{C}_{\text {gen }}(\tau)=\max _{\partial \Sigma(\tau)=\Sigma_{\tau}}\left[\frac{1}{G_{N} L} \int_{\Sigma} d^{d} \sigma \sqrt{h} F_{1}\left[g_{\mu \nu} ; X^{\mu}(\sigma)\right]\right]$.

Because of the symmetries of the state and the hypersurface $\Sigma(\tau)$, we can parametrize our surface with a single

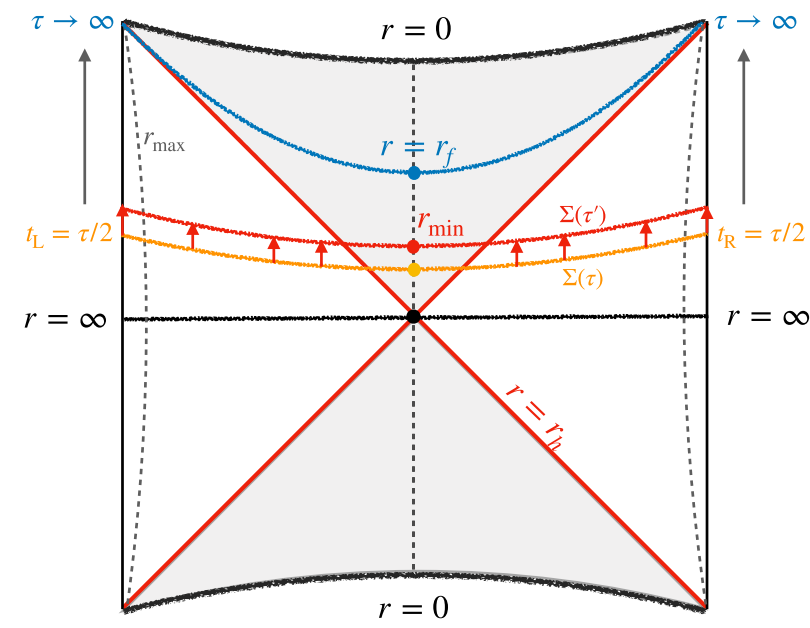

FIG. 1. The time evolution of the extremal hypersurfaces from $\Sigma(\tau)$ to a nearby extremal hypersurface $\Sigma\left(\tau^{\prime}\right)$. At infinite time limit $\tau \rightarrow \infty$, the extremal surface approaches a constant $r$ hypersurface at $r=r_{f}$, where the effective potential arrives at a local maximal value. 
function $r(v)$. When $F_{1}=F_{2}$, finding the extremal surface is analogous to solving a classical mechanics problem where the action and Lagrangian are identified as $S \sim \mathcal{C}_{\text {gen }}$, $\mathcal{L} \sim F_{1} \sqrt{h}$. Moreover, the extremality conditions become the equations of motion derived from $\mathcal{L}$.

In this picture, the time derivative of $\mathcal{C}_{\text {gen }}$ evaluated on the extremal surface is related to the momentum at the end point of the trajectory:

$$
\frac{d \mathcal{C}_{\text {gen }}}{d \tau}=\left.\left.\frac{1}{2} \mathcal{P}_{t}\right|_{\partial \Sigma(\tau)} \equiv \frac{1}{2} \frac{\partial \mathcal{L}}{\partial \dot{t}}\right|_{\partial \Sigma(\tau)},
$$

where $\mathcal{P}_{t}$ is the momentum conjugate to the coordinate time $t$. Linear growth amounts to the statement that $\left.\mathcal{P}_{t}\right|_{\partial \Sigma(\tau)}$ approaches a constant at large $\tau$.

Time evolution of the extremal surfaces.-We will now proceed to solve for the location of the extremal surfaces as a function of time and study the late time behavior of our new observables. Because of the symmetries of the planar black hole, we can parametrize the spacelike hypersurface $\Sigma$ simply by $[v(\sigma), r(\sigma), \vec{x}]$. Derivatives with respect to $\sigma$ will be represented by dots. In this Letter, we will focus on observables where the function $F_{1}\left(g_{\mu \nu}, \mathcal{R}_{\mu \nu \rho \sigma}, \nabla_{\mu}\right)$ depends only on $(d+1)$-dimensional curvature invariants evaluated on the extremal slice $\Sigma$. (We comment on more general observables in the discussion.) For such observables, one can rewrite the generalized volume in our parametrization by

$$
\mathcal{C}_{\text {gen }}=\frac{V_{x}}{G_{N} L} \int_{\Sigma} d \sigma\left(\frac{r}{L}\right)^{d-1} \sqrt{-f(r) \dot{v}^{2}+2 \dot{v} \dot{r}} a(r),
$$

where $\sigma$ can be understood as a radial coordinate on the hypersurface $\Sigma, V_{x}$ denotes the (regulated) volume of the spatial boundary directions $\vec{x}$, and the factor $a(r)$ is the result of evaluating the corresponding function of curvatures on the surface. The restriction to $(d+1)$ dimensional curvature invariants was made so that the factor $a(r)$ does not depend on derivatives of $r$, which considerably simplifies the problem [10].

Of course, we also require that the functionals $\mathcal{C}_{\text {gen }}$ are diffeomorphism invariant. Consequently, one can easily show that $\mathcal{C}_{\text {gen }}$ is invariant under the transformation $\sigma \rightarrow g(\sigma)$. As a result, we can fix the gauge by choosing

$$
\sqrt{-f(r) \dot{v}^{2}+2 \dot{v} \dot{r}}=a(r)\left(\frac{r}{L}\right)^{d-1} .
$$

Because the bulk spacetime is stationary, one finds that the momentum $P_{v}$ conjugate to the infalling time $v$, i.e.,

$$
P_{v}=\frac{a(r)(r / L)^{d-1}[\dot{r}-f(r) \dot{v}]}{\sqrt{-f(r) \dot{v}^{2}+2 \dot{v} \dot{r}}}=\dot{r}-f(r) \dot{v},
$$

is conserved on the extremal surfaces. Combining these two equations, one arrives at the extremality conditions:

$$
\begin{aligned}
& \dot{r}= \pm \sqrt{P_{v}^{2}+f(r) a^{2}(r)\left(\frac{r}{L}\right)^{2(d-1)}}, \\
& \dot{v}=\frac{1}{f(r)}\left(-P_{v} \pm \sqrt{P_{v}^{2}+f(r) a^{2}(r)\left(\frac{r}{L}\right)^{2(d-1)}}\right) .
\end{aligned}
$$

We can recast this problem as the motion of a classical nonrelativistic particle in a potential $[11,12]$. To wit,

$\dot{r}^{2}+\tilde{U}(r)=P_{v}^{2} \quad$ with $\quad \tilde{U}(r)=-f(r) a^{2}(r)\left(\frac{r}{L}\right)^{2(d-1)}$,

with an effective potential $\tilde{U}$ which parametrizes the choice of observable. Figure 2 presents a characteristic potential. Because of the multiplicative prefactor $f(r)$, the effective potential vanishes at the horizon $r=r_{h}$. We will particularly be interested in the case where the effective potential has at least one local maximum inside the horizon. As we will show, this is precisely the requirement for the linear growth of the generalized volume. When the couplings for the higher curvature terms in $F_{1}$ are sufficiently small, such a local maximum always exists [10]. We can specify the local maximum at $r=r_{f}$ by

$$
\tilde{U}\left(r_{f}\right)=P_{\infty}^{2}, \quad \tilde{U}^{\prime}\left(r_{f}\right)=0, \quad \tilde{U}^{\prime \prime}\left(r_{f}\right) \leq 0 .
$$

Finding the extremal surface anchored on a specific boundary time slice $\tau$ thus corresponds to solving the

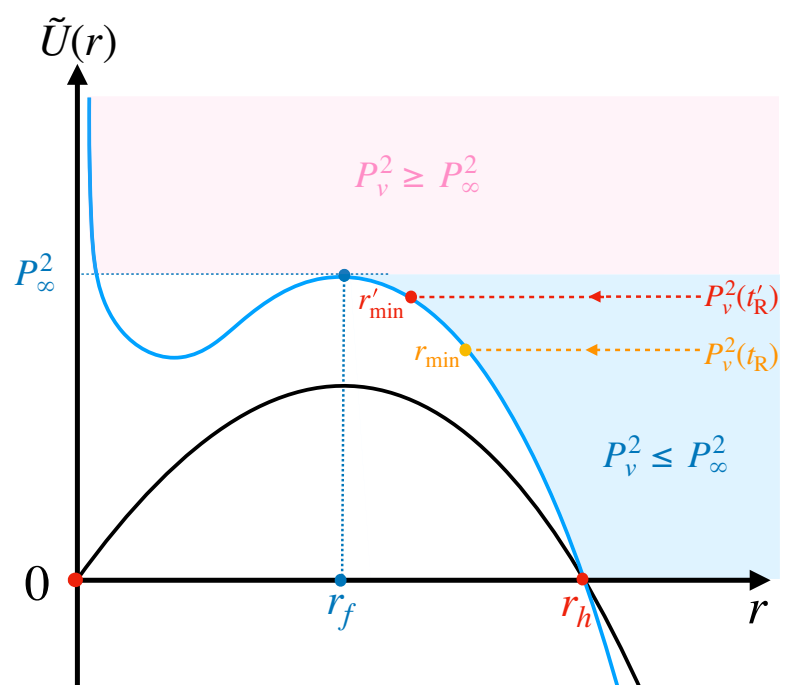

FIG. 2. The blue curve denotes a characteristic effective potential $\tilde{U}(r)$ depending on the spacetime curvature and the black curve presents the potential from the volume. The turning point at the minimal radius $r_{\min }$ satisfies $\dot{r}=0$, which is equivalent to $P_{v}^{2}=\tilde{U}\left(r_{\min }\right)$ for a given conserved momentum. The critical value of the momentum $P_{\infty}$ is obtained at the infinite boundary time $\tau \rightarrow \infty$. 
Hamiltonian system (12) with a given conserved momentum $P_{v}$ that is fixed by the boundary time, namely [13],

$$
\tau \equiv 2 t_{R}=-2 \int_{r_{\min }}^{\infty} d r \frac{P_{v}}{f(r) \sqrt{P_{v}^{2}-\tilde{U}(r)}} .
$$

We denote $r_{\min }$ as the minimal radius lying on the timelike surface $t=0$ (dashed vertical line in Fig. 1) and determined by the conserved momentum through $P_{v}^{2}=\tilde{U}\left(r_{\min }\right)$. This is also the turning point of the analog particle. As discussed in Eq. (7), the time derivative of the generalized volume at boundary time $\tau$ is given by

$$
\frac{d \mathcal{C}_{\text {gen }}}{d \tau}=\left.\frac{1}{2} \mathcal{P}_{t}\right|_{\partial \Sigma}=\frac{V_{x}}{G_{N} L} P_{v}(\tau)
$$

Linear growth at late times.-For any boundary time $\tau$, fixing the conserved momentum $P_{v}(\tau)$ via Eq. (14), there is a corresponding extremal hypersurface. For example, $P_{v}^{2}=0$ gives the time reflection symmetric slice $t=0$. For generic $\tau$, the radial trajectory of the extremal surface starts from the asymptotic boundary and moves into the interior of the black hole until it reaches the minimal radius $r_{\text {min }}$. Increasing the conserved momentum $P_{v}$ to the critical value $P_{\infty}$ causes the boundary time to diverge because the extremal surface is near the final slice at $r=r_{f}$. This can be seen by expanding the potential around the maximum

$P_{\infty}^{2}-\tilde{U}(r) \sim-\frac{1}{2} \tilde{U}^{\prime \prime}\left(r_{f}\right)\left(r-r_{f}\right)^{2}+\mathcal{O}\left[\left(r-r_{f}\right)^{3}\right]$,

and substituting into Eq. (14). Then we note that a divergence arises from the lower boundary of the integral as $r_{\min }$ approaches $r_{f}$. Similarly, we will now demonstrate that $\lim _{\tau \rightarrow \infty} P_{v}(\tau)=P_{\infty}$. Because of Eq. (15), this means that the generalized volume grows linearly at late times. Before doing so, we stress again that the existence of the above late-time limit requires the existence of a local maximum of the effective potential inside the horizon.

Using Eq. (14), it is straightforward to get

$$
\begin{aligned}
\frac{d \tau}{d P_{v}}= & \left.\frac{d r_{\min }}{d P_{v}} \frac{2 P_{v}}{f(r) \sqrt{P_{v}^{2}-\tilde{U}(r)}}\right|_{r \rightarrow r_{\min }} \\
& +\int_{r_{\min }}^{\infty} d r \frac{2 \tilde{U}(r)}{f(r)\left[P_{v}^{2}-\tilde{U}(r)\right]^{3 / 2}},
\end{aligned}
$$

where both terms on the right-hand side are divergent due to $P_{v}^{2}-\tilde{U}\left(r_{\min }\right)=0$. Approaching the infinite boundary time by pushing the minimal radius to the final slice (i.e., $r_{\text {min }} \rightarrow r_{f}$ or, equivalently, $\left.P_{v} \rightarrow P_{\infty}\right)$, the leading divergence appearing in Eq. (17) in the late-time limit is

$$
\frac{d \tau}{d P_{v}} \sim \frac{-2 \sqrt{2} \tilde{U}\left(r_{f}\right)}{f\left(r_{f}\right)\left[-\tilde{U}^{\prime \prime}\left(r_{f}\right)\right]^{\frac{3}{2}}} \frac{1}{\left(r_{\min }-r_{f}\right)^{2}} .
$$

More explicitly, the above limit implies that in the late-time limit, the growth rate $P_{v}(\tau)$ approaches the critical value $P_{\infty}$ from below as an exponential

$P_{\infty}-P_{v}(\tau) \propto e^{-\kappa \tau}, \quad$ with $\quad \kappa=\frac{-f\left(r_{f}\right) \sqrt{-2 \tilde{U}^{\prime \prime}\left(r_{f}\right)}}{P_{\infty}}$.

Note that $\kappa$ is always positive, since the final slice is inside the horizon [hence $f\left(r_{f}\right)<0$ ] and corresponds to a local maximum of the potential with $\tilde{U}^{\prime \prime}\left(r_{f}\right)<0$.

Finally, let us show that $P_{\infty} \propto M$ where $M \propto\left(r_{h}^{d} / G_{N} L^{2}\right)$ is the ADM mass of the black brane. We can introduce a dimensionless radial coordinate $w=\left(r / r_{h}\right)^{d}$, in terms of which the potential is rewritten as

$$
\tilde{U}(r)=\left(\frac{r_{h}}{L}\right)^{2 d}\left(w-w^{2}\right) a^{2}(w) .
$$

We note that $a(w)$ depends only on $w$ because curvature invariants in the geometry (5) are independent of $r_{h}$ when written as a function of $w$. This is easy to see by using rescaled coordinates $\left(r_{h} / L\right) v,\left(r_{h} / L\right) \vec{x}$, in which metric components have no dependence on $r_{h}$. The dimensionful coefficients appearing in the definition of $F_{1}$ should not depend on $r_{h}$ either, because we want to define the observable in a state independent way. Using the recast potential (20), it is easy to show that its extrema satisfies

$$
P_{\infty}^{2} \equiv \tilde{U}\left(r_{f}\right)=\left(\frac{r_{h}}{L}\right)^{2 d} \xi
$$

where the constant $\xi$ is independent of $r_{h}$ [14].

More general observables with $F_{1} \neq F_{2}$.-We can also consider observables that are associated with the function $F_{1}$ but are evaluated on the extremal surface determined by the functional $F_{2}$. Let us focus on the simplest case where both $F_{1}$ and $F_{2}$ only depend on the bulk spacetime geometry. To wit, we define the observable as

$O_{F_{1}, \Sigma_{F_{2}}} \propto \int_{\Sigma_{F_{2}}} d \sigma\left(\frac{r}{L}\right)^{d-1} \sqrt{-f(r) \dot{v}^{2}+2 \dot{v} \dot{r}} a_{1}(r)$,

where the extremal hypersurfaces $\Sigma_{F_{2}}$ are associated with the effective potential $\tilde{U}_{2}(r)=-f(r) a_{2}^{2}(r)(r / L)^{2(d-1)}$. For $F_{1}=F_{2}$, the time derivative was given by the boundary term $P_{v}$. Now, the time derivative of this new observable with respect to the boundary time $\tau$ reads instead

$$
\begin{aligned}
\frac{G_{N} L}{V_{x}} \frac{d O_{F_{1}, \Sigma_{F_{2}}}}{d \tau}= & \sqrt{\bar{U}_{1}}+P_{v} \frac{d P_{v}}{d \tau} \int_{r_{\min }}^{\infty} d r \\
& \times \frac{\sqrt{\tilde{U}_{1}(r) \tilde{U}_{2}(r)}-\sqrt{\frac{\bar{U}_{1}}{\bar{U}_{2}}} \tilde{U}_{2}(r)}{f(r)\left[P_{v}^{2}-\tilde{U}_{2}(r)\right]^{3 / 2}},
\end{aligned}
$$


where we defined $\bar{U}_{i}=\bar{U}_{i}\left(r_{\text {min }}\right)$ and the second term involving the integral vanishes when $\tilde{U}_{1}=\tilde{U}_{2}$. As with the analysis above, we find the new observable (22) still exhibits the expected linear growth at late times, i.e.,

$$
\lim _{\tau \rightarrow \infty} \frac{d O_{F_{1}, \Sigma_{F_{2}}}}{d \tau}=\frac{V_{x}}{G_{N} L} \sqrt{\tilde{U}_{1}\left(r_{f}\right)},
$$

where $r_{f}$ is the position of the maximum of $\tilde{U}_{2}(r)$. But the growth rate is controlled by the functional $F_{1}$ [10].

Switchback effect.-Consider the perturbed TFD state

$$
\left|\Psi\left(t_{L}, t_{R}\right)\right\rangle=e^{-i H_{L} t_{L}-i H_{R} t_{R}} W_{L}\left(t_{n}\right) \ldots W_{L}\left(t_{1}\right)\left|\psi_{\mathrm{TFD}}(0)\right\rangle,
$$

where the $t_{1}, \ldots, t_{n}$ are in an alternating "zig-zag" order. The corresponding complexity is then proportional to [9]

$$
\left|t_{R}+t_{1}\right|+\left|t_{2}-t_{1}\right|+\cdots+\left|t_{L}-t_{n}\right|-2 n t_{*} .
$$

Here, the terms with absolute values give the total length of the path integral contour. The switchback effect is the subtraction $-2 n t_{*}$, owing to the partial cancellation of forward and backward parts of the time contours around the "switchbacks," where the operator insertions have not been scrambled yet. The formula assumes that the time along each switchback (i.e., each term involving an absolute value) is much larger than the scrambling time.

The state (25) is dual to a long wormhole supported by alternating left- and right-moving shock waves. When all shocks are strong (which we study here), the geometry is obtained by gluing together AdS black hole geometries (5) along their horizons [15]. There are $n+1$ such patches, and the wormhole region consists of $n-1$ future and past interiors glued together in an alternating manner. The gluing is best described in Kruskal coordinates

$$
U \cdot V=-e^{f^{\prime}\left(r_{h}\right) r_{*}(r)}, \quad \frac{U}{V}=-e^{-f^{\prime}\left(r_{h}\right) t},
$$

in which the corresponding null shifts along the horizons are given by $\alpha_{i}=2 e^{-(2 \pi / \beta)\left(t_{*} \pm t_{i}\right)}$ with the inverse temperature $\beta=\left[4 \pi / f^{\prime}\left(r_{h}\right)\right]$ and the scrambling time $t_{*} \propto \beta \log G_{N}^{-1}$.

The switchback effect associated with the maximal volume in this geometry was analyzed in Ref. [9]. It is based on two properties of the extremal volume: (i) that it adds up in shock wave geometries, i.e.,

$$
\begin{aligned}
\mathcal{V}= & \mathcal{V}\left(t_{R}, V_{1}\right)+\mathcal{V}\left(V_{1}+\alpha_{1}, U_{2}\right)+\cdots \\
& +\mathcal{V}\left(U_{n-1}-\alpha_{n-1}, V_{n}\right)+\mathcal{V}\left(V_{n}+\alpha_{n}, t_{L}\right),
\end{aligned}
$$

where $\mathcal{V}(.,$.$) denotes the volume of the extremal slice$ connecting two specified points (either $V_{i}, U_{i}$ on a horizon or $t_{L, R}$ on the boundary) in the black hole geometry; (ii) that
$\mathcal{V}(.,$.$) all present a linear growth at late times. In order$ to find the maximal volume slice, one needs to extremize the additive volume in Eq. (28) with respect to $V_{1}$, $U_{2}, \ldots U_{n-1}, V_{n}$ which leads to the result (26).

We find that these two properties are also shared by the general observables $O_{F_{1}, \Sigma_{F_{2}}}$ defined in Eq. (22). The property (i) could in general fail when the functional involves curvature terms, since there could be extra $\delta$-function contributions at the shock waves, spoiling the additivity property. However, we can prove that the diffeomorphism invariance of the functional $F_{1}$ guarantees that no contribution arises from $\delta$ functions on the shock waves. Consider the geometry (5) in Kruskal coordinates

$$
d s^{2}=-2 A(U V) d U d V+B(U V) d \vec{x}^{2},
$$

where $A=-(2 / U V)\left[f(r) / f^{\prime}\left(r_{h}\right)^{2}\right], B=r^{2} / L^{2}$ are both functions of $U V$ because the spacetime is stationary. For simplicity, we consider a single null shock wave at $U=0$. The backreaction on the geometry is described by $[9,15,16]$

$$
\begin{aligned}
d s^{2}= & -2 A(U[V+\alpha \Theta(U)]) d U d V \\
& +B(U[V+\alpha \Theta(U)]) d \vec{x}^{2},
\end{aligned}
$$

where $\Theta(U)$ denotes the Heaviside step function. Suppose we want to write down a scalar function $F_{1}$ which only depends on the bulk geometry. All metric components depend on $U$ and $V$ as $f(U[V+\alpha \Theta(U)])$. Therefore, delta functions can come only from $U$ derivatives of metric components. Since $g_{U U}=g_{V V}=0$, in order to form scalars we must eventually contract all indices coming from derivatives with metric components $g^{U V}$, e.g., $g^{U V} \partial_{V} \partial_{U} f$. As a result, one can show that all $U$ derivatives must come multiplied by an equal number of $V$ derivatives in scalar functions. Noting that $\partial_{V} f(U[V+\alpha \Theta(U)])=$ $U f^{\prime}(U[V+\alpha \Theta(U)])$, we find that delta functions always come in the form $U \delta(U), U^{2} \delta^{\prime}(U)$, etc. Since in the Kruskal geometry the functions $A$ and $B$ are regular and nonzero on the horizon we conclude that no delta functions appear in spacetime scalars formed from the shock wave geometry [17].

Regarding the property (ii), we can show following section 4 that the general observables $O_{F_{1}, \Sigma_{F_{2}}}$ are still dominated by linear growth in ingoing and outgoing time, i.e.,

$O_{F_{1}, \Sigma_{F_{2}}}\left(U_{L}, V_{R}\right)=\frac{V_{x}}{G_{N} L} \sqrt{\tilde{U}_{1}\left(r_{f}\right)}\left|v_{R}+u_{L}\right|+O(1)$,

where $\tilde{U}_{1}\left(r_{f}\right)$ denotes the value of the effective potential on the final slice and the Kruskal coordinate is given by $|U|=e^{-(2 \pi / \beta) u},|V|=e^{(2 \pi / \beta) v}$. Using the additive formula in Eq. (28) for $O_{F_{1}, \Sigma_{F_{2}}}$ and extremizing with respect to $V_{1}, U_{2}, \ldots U_{n-1}, V_{n}$, one recovers the expected switchback effect (26). 
Discussion.-In this Letter, we have introduced an infinite family of gravitational observables $O_{F_{1}, \Sigma_{F_{2}}}$ defined on codimension-one slices of the geometry. We examined a large class of such observables, where the functionals $F_{1}$, $F_{2}$ only depend on spacetime curvature invariants, and showed they display universal behavior: in the timeevolved thermofield double state, they grow linearly in time at late times and exhibit the switchback effect. However, we note that this universal behavior also requires the couplings on the curvature invariants not be too large. More general observables including functionals that depend independently on extrinsic and intrinsic data of the surface will be discussed in Ref. [19], and we expect our results to extend to these cases as well. We now conclude with some open questions.

In light of the CA proposal, a natural question is to ask whether it is also possible to engineer codimension-0 observables like the action of the Wheeler-de Witt patch. In fact, it is possible to embed both codimension- 0 and codimension-one observables in a unified framework following a construction by Peierls [20]. This will be presented in Ref. [19], where we will also discuss how to extract variations of these observables from the CFT, for coherent states of the gravitational theory [21-26] using the dictionary between bulk and boundary symplectic forms.

Finally, we have given strong evidence that a wide class of observables are all viable candidates for complexity. It would be interesting to try and make the connection between ambiguities in the definition of quantum complexity, and the choice of gravitational observables more precise. However, is there perhaps a reason that would single out the volume? The maximal volume not only captures the saturation of complexity at very late times [27] but also satisfies nicer properties like existence theorems for the maximal slice [28], positivity of the vacuum-subtracted volume [29], regular behavior under small deformations [30] and serving as an internal Hamiltonian of the Wheelerde Witt patch $[24,25]$. It would be interesting to explore this question further in the future.

We are happy to thank Jan de Boer, Shuwei Liu, Tadashi Takayanagi, and Michael Walter for fruitful discussions and useful comments. Research at Perimeter Institute is supported in part by the Government of Canada through the Department of Innovation, Science and Economic Development Canada and by the Province of Ontario through the Ministry of Colleges and Universities. R. C. M. is supported in part by a Discovery Grant from the NSERC of Canada, and by the BMO Financial Group. R.C.M. also received funding from the Simons Foundation through the "It from Qubit" collaboration. A. J. S. is supported by the Air Force Office of Scientific Research under Award No. FA9550-19-1-036. *a.belin@cern.ch

rmyers@perimeterinstitute.ca

*sruan@perimeterinstitute.ca

§abor.sarosi@cern.ch

" asperanz@gmail.com

[1] T. Hartman and J. Maldacena, Time evolution of entanglement entropy from black hole interiors, J. High Energy Phys. 05 (2013) 014.

[2] L. Susskind, Computational complexity and black hole horizons, Fortschr. Phys. 64, 44 (2016); 64, 24 (2016).

[3] A. R. Brown, D. A. Roberts, L. Susskind, B. Swingle, and Y. Zhao, Holographic Complexity Equals Bulk Action?, Phys. Rev. Lett. 116, 191301 (2016).

[4] J. Couch, W. Fischler, and P. H. Nguyen, Noether charge, black hole volume, and complexity, J. High Energy Phys. 03 (2017) 119.

[5] L. Lehner, R. C. Myers, E. Poisson, and R. D. Sorkin, Gravitational action with null boundaries, Phys. Rev. D 94, 084046 (2016).

[6] M. Alishahiha, Holographic complexity, Phys. Rev. D 92, 126009 (2015).

[7] P. Bueno, V. S. Min, A. J. Speranza, and M. R. Visser, Entanglement equilibrium for higher order gravity, Phys. Rev. D 95, 046003 (2017).

[8] J. Hernandez, R. C. Myers, and S.-M. Ruan, Quantum extremal islands made easy. Part III. Complexity on the brane, J. High Energy Phys. 02 (2021) 173.

[9] D. Stanford and L. Susskind, Complexity and shock wave geometries, Phys. Rev. D 90, 126007 (2014).

[10] See Supplemental Material at http://link.aps.org/ supplemental/10.1103/PhysRevLett.128.081602 for Appendix A containing a detailed analysis involving a Weyl-squared term $C_{\mu \nu \rho \sigma} C^{\mu \nu \rho \sigma}$, and Appendix B containing more details on the linear growth of observables with $F_{1} \neq F_{2}$.

[11] D. Carmi, S. Chapman, H. Marrochio, R. C. Myers, and S. Sugishita, On the time dependence of holographic complexity, J. High Energy Phys. 11 (2017) 188.

[12] S. Chapman, H. Marrochio, and R. C. Myers, Holographic complexity in Vaidya spacetimes. Part II, J. High Energy Phys. 06 (2018) 114.

[13] We note that the integrand is divergent at the horizon because $f(r) \sim f^{\prime}\left(r_{h}\right)\left(r-r_{h}\right)$. Hence this integral (14) is defined by the Cauchy principal value associated with this singularity, which is finite.

[14] This result relies on having a scale invariant planar horizon, corresponding to a thermodynamic limit in the CFT. For compact horizons, there are finite size corrections, just like in the case of the CV proposal [11].

[15] S. H. Shenker and D. Stanford, Multiple Shocks, J. High Energy Phys. 12 (2014) 046.

[16] K. Sfetsos, On gravitational shock waves in curved spacetimes, Nucl. Phys. B436, 721 (1995).

[17] Clearly, this result will also hold for more general stationary spacetimes, e.g., black holes with spherical or hyperbolic horizons. One can view this as a generalization of a similar result [18] for AdS shock waves to static Kruskal geometries. 
[18] G. T. Horowitz and N. Itzhaki, Black holes, shock waves, and causality in the AdS/CFT correspondence, J. High Energy Phys. 02 (1999) 010.

[19] A. Belin, R. C. Myers, S.-M. Ruan, G. Sárosi, and A. J. Speranza (to be published).

[20] R. E. Peierls, The Commutation laws of relativistic field theory, Proc. R. Soc. A 214, 143 (1952).

[21] M. Botta-Cantcheff, P. Martinez, and G. A. Silva, On excited states in real-time AdS/CFT, J. High Energy Phys. 02 (2016) 171.

[22] D. Marolf, O. Parrikar, C. Rabideau, A. Izadi Rad, and M. Van Raamsdonk, From Euclidean sources to Lorentzian spacetimes in holographic conformal field theories, J. High Energy Phys. 06 (2018) 077.

[23] A. Belin, A. Lewkowycz, and G. Sarosi, The boundary dual of the bulk symplectic form, Phys. Lett. B 789, 71 (2019).

[24] A. Belin, A. Lewkowycz, and G. Sarosi, Complexity and the bulk volume, a New York time story, J. High Energy Phys. 03 (2019) 044.
[25] A. Belin and B. Withers, From sources to initial data and back again: On bulk singularities in Euclidean AdS/CFT, J. High Energy Phys. 12 (2020) 185.

[26] A. Bernamonti, F. Galli, J. Hernandez, R. C. Myers, S.-M. Ruan, and J. Simón, First Law of Holographic Complexity, Phys. Rev. Lett. 123, 081601 (2019).

[27] L. V. Iliesiu, M. Mezei, and G. Sárosi, The volume of the black hole interior at late times, arXiv:2107.06286.

[28] E. Witten, Canonical quantization in anti de Sitter space, in PCTS (2017), http://www.kaltura.com/index.php/extwidget/ preview/partner_id/1449362/uiconf_id/14292322/entry_id/ 1_iy6rwyi1/embed/auto?\&flashvars\%5BstreamerType $\% 5 \mathrm{D}=$ auto.

[29] N. Engelhardt and R. Folkestad, General bounds on holographic complexity, J. High Energy Phys. 01 (2022) 040.

[30] M. Flory, Discrete scale invariance in holography and an argument against the complexity $=$ action proposal, Acta Phys. Pol. B Proc. Suppl. 13, 267 (2020). 